Pacific Journal of Mathematics

LINES HAVING HIGH CONTACT WITH A PROJECTIVE 


\section{LINES HAVING HIGH CONTACT WITH A PROJECTIVE VARIETY}

\section{GEORGE JENNINGS}

Let $\mathscr{U}$ open $\subset \mathbf{P}^{n}=\mathbf{P}^{n}(C), X \subset \mathscr{U}$ an analytic subvariety,

$$
\begin{array}{rl}
J & =\left\{(p, l) \in \mathbf{P}^{n} \times \mathbf{G}(1, n) \mid p \in l\right\} \\
\pi \swarrow & \searrow \lambda \\
\mathbf{P}^{n} & \mathbf{G}=\mathbf{G}(1, n),
\end{array}
$$

the incidence correspondence with induced projections $\pi, \lambda$, where $\mathbf{G}=\mathbf{G}(1, n)$ is the Grassmannian of lines in $\mathbf{P}^{n}$.

0. Definition. The contact cones of $X$ are

$$
\begin{aligned}
C^{r} & =\left\{(p, l) \in \pi^{-1} \mathscr{U} \mid l \text { has contact } \geq r+1 \text { with } X \text { at } p\right\} \\
C^{\infty} & =\bigcap_{r=0}^{\infty} C^{r} .
\end{aligned}
$$

The contact cones may be thought of as schemes of cones in the tangent space of $\mathbf{P}^{n}$ which reflect the local geometry of the embedding $X \rightarrow \mathscr{U}$. The main results of this paper are a singularities theorem (13) which puts an upper bound on the pathology of the contact cones if $X$ is not ruled, and an algebraization theorem (17) which says roughly that if $X$ is a hypersurface whose contact cones resemble those of an algebraic hypersurface of low degree then $X$ is algebraic. Hypersurfaces are the simplest case - in a future paper we show that in general hypersurfaces are determined up to projective equivalence by the projective moduli of the third contact cone with a little help from the ideal of the fourth.

The contact cones have a scheme structure defined in terms of the functor of principal parts (jets) $\mathscr{P}_{J / G}^{r}[5, \S 16]$. Let $\mathscr{F}$ be a sheaf of $\mathcal{O}_{J}$-modules. Form the fiber product $J \times_{\mathbf{G}} J$. Let $\mathscr{I}_{\Delta}$ be the ideal sheaf of the diagonal, and $J^{r} \stackrel{\Delta^{r}}{\rightarrow} J \times_{\mathbf{G}} J$ the subscheme defined by $\mathscr{I}_{\Delta}^{r+1}$. One has a commutative diagram

$$
\begin{array}{ccccc} 
& J^{r} & & \\
& p^{r} \swarrow & \downarrow \Delta^{r} & \searrow q^{r} & \\
J & \stackrel{p}{\leftarrow} & J \times_{\mathbf{G}} J & \stackrel{q}{\rightarrow} & J
\end{array}
$$


where $p, q$ are the projections. Then

$$
\mathscr{P}_{J / \mathbf{G}}^{r} \mathscr{F}=q_{*}^{r} p^{r * \mathscr{F}} \cong \mathscr{F} \otimes_{\mathcal{O}_{J}} \mathscr{P}_{J / \mathbf{G}}^{r} \mathcal{O}_{J}
$$

$\mathscr{P}_{J / \mathbf{G}}^{r} \mathcal{O}_{J}$ is a locally free sheaf of rank $r+1$ consisting of relative $r$ jets of sections of $\mathcal{O}_{J}$.

Let $\mathscr{I}_{X} \subset \mathcal{O}_{\mathscr{U}}$ be the ideal sheaf of $X$.

1. Definition. $C^{r}, 0 \leq r<\infty$, is the zero scheme of the sheaf of sections $\mathscr{P}_{J / \mathbf{G}}^{r}\left(\pi^{*} \mathscr{I}_{X}\right) \subset \mathscr{P}_{J / G}^{r} \mathcal{O}_{J} \mid \mathscr{U} . C^{\infty}=\bigcap_{r=0}^{\infty} C^{r}$ is the intersection scheme. $C_{p}^{r}=\pi^{-1}(p) \cap C^{r}, 0 \leq r \leq \infty$, is the fiber over $p \in \mathscr{U}$.

Since $J \times_{\mathbf{G}} J \stackrel{\pi \times \pi}{\rightarrow} \mathbf{P}^{n} \times \mathbf{P}^{n}$ is the blow up of $\mathbf{P}^{n} \times \mathbf{P}^{n}$ along the diagonal the exceptional divisor $J$ is naturally isomorphic to the projectivized tangent space $P T \mathbf{P} \rightarrow \mathbf{P}^{n}$, via the relation " $v$ is tangent to $l$ ". In particular the relative cotangent sheaf $\Omega_{J / \mathbf{G}}^{1} \cong \mathscr{I}_{\Delta} / \mathscr{I}_{\Delta}^{2}$ of $J$ is just the dual $\mathcal{O}_{T}(1)$ of the universal subbundle $\mathcal{O}_{T}(-1)$ of $\pi^{*} T \mathbf{P}$ over PTP. $J \cong$ $\operatorname{Proj}\left(S \cdot \Omega_{J / \mathbf{G}}^{1} \mathbf{P}\right)$ where $S \cdot \Omega_{J / \mathbf{G}}^{1}$ is the sheaf of graded rings

$$
S \cdot \Omega_{J / \mathbf{G}}^{1} \cong \mathcal{O}_{J} \oplus \mathscr{I}_{\Delta} / \mathscr{I}_{\Delta}^{2} \oplus \mathscr{I}_{\Delta}^{2} / \mathscr{I}_{\Delta}^{3} \oplus \cdots .
$$

There is an (additive) sheaf homomorphism $d_{J / G}^{r}: \mathcal{O}_{J} \rightarrow \mathscr{P}_{J / G}^{r} \mathcal{O}_{J}$ induced by the corresponding map on sections [5, p. 16]. One has a commutative diagram

$$
\begin{array}{lcccccc} 
& d_{J / \mathbf{G}}^{r}\left(\pi^{*} \mathscr{I}_{X}\right) & \rightarrow & d_{J / \mathbf{G}}^{r-1}\left(\pi^{*} \mathscr{I}_{X}\right) & \rightarrow & 0 \\
& & \downarrow & & \downarrow & & \\
0 \rightarrow \mathscr{I}_{\Delta}^{r} / \mathscr{I}_{\Delta}^{r+1} & \stackrel{\iota}{\rightarrow} & \mathscr{P}_{J / \mathbf{G}}^{r} \mathcal{O}_{J} & \stackrel{\rho}{\rightarrow} & \mathscr{P}_{J / \mathbf{G}}^{r-1} \mathcal{O}_{J} & \rightarrow & 0
\end{array}
$$

over $\pi^{-1} \mathscr{U}$ arising directly from the definition. Define contact ideal sheaves $\left.\mathscr{J}_{X}^{r} \subset S \Omega_{J / \mathbf{G}}^{1}\right|_{\pi^{-1} \mathscr{U}}$ inductively by

$$
\begin{aligned}
& \mathscr{J}_{X}^{0}=\pi^{*} \mathscr{I}_{X} \otimes_{\mathcal{O}_{J}} S \cdot \Omega_{J / \mathbf{G}}^{1} \\
& \mathscr{J}_{X}^{r}=\mathscr{J}_{X}^{r-1}+\iota^{-1}\left(d_{J / \mathbf{G}}^{r}\left(\pi * \mathscr{I}_{X}\right)+\operatorname{ker} \rho\right) \otimes_{\mathcal{O}_{J}} S \cdot \Omega_{J / \mathbf{G}}^{1} \\
& \mathscr{J}_{X}^{\infty}=\sum_{r=0}^{\infty} \mathscr{J}_{X}^{r}
\end{aligned}
$$

on $\pi^{-1} \mathscr{U} . \mathscr{J}_{X}^{r}$ is the ideal sheaf of $C^{r}$ in $S \Omega_{J / \mathbf{G}}^{1}$.

This leads to a convenient version in coordinates. Let $x=\left(x_{1}, \ldots, x_{n}\right)$ be an affine coordinate system on $\mathscr{U}, d x=\left(d x_{1}, \ldots, d x_{n}\right), p \in \mathscr{U}, g \in$ $\mathcal{O}_{p, \mathscr{U}}$. Expand in a power series

$$
g(x(p)+t)=g^{0}(x)+g^{1}(x ; t)+g^{2}(x ; t)+\cdots
$$


where $t=\left(t_{1}, \ldots, t_{n}\right)$ are indeterminants and $g^{r}(x ; t)$ is the $r$ th order term. Replacing $t$ by $d x$,

$$
\mathscr{J}_{X}^{r}=\left(g^{s}(x ; d x) \mid 0 \leq s \leq r, g \in \mathscr{I}_{X}\right)
$$

in coordinates.

The geometry of the contact cones is controlled by the "derivative relation":

$$
\frac{\partial g^{r}(x ; t)}{\partial x_{i}}=\frac{\partial g^{r+1}(x ; t)}{\partial t_{i}}, \quad i=1, \ldots, n,
$$

in coordinates (see [5, p. 43] for a coordinate free version).

12. Proposition. If $\mathscr{J}_{X}^{r}=\mathscr{J}_{X}^{r+1}$ for some $r$ then $\mathscr{J}_{X}^{r}=\mathscr{J}_{X}^{\infty}$, so $\pi C^{r} \subset$ $X$ is ruled (by line segments).

Proof. Let $g_{1}, \ldots, g_{m}$ generate $\mathscr{I}_{X}$ over $\mathscr{U}$. By hypothesis there exists a local relation

$$
g_{i}^{r+1}(x ; t)=\sum_{j=1}^{m} \sum_{s=0}^{r} a_{i j s}(x ; t) g_{j}^{s}(x ; t), \quad i=1, \ldots, m .
$$

Differentiate with respect to $x_{\lambda}$ and apply the derivative relation (11):

$$
\frac{\partial g_{i}^{r+2}}{\partial t_{\lambda}} \equiv \sum_{j=1}^{m} \sum_{s=0}^{r} a_{i j s} \frac{\partial g_{j}^{s+1}}{\partial t_{\lambda}} \bmod \mathscr{J}_{X}^{r}
$$

$\lambda=1, \ldots, n$. Multiply by $t_{\lambda}$, sum over $\lambda$, and apply the Euler relation:

$$
(r+2) g^{r+2} \equiv 0 \quad \bmod \mathscr{J}_{X}^{r+1} \text {. }
$$

Continue inductively. Since $\pi C^{\infty}$ is obviously ruled we are done. (Of course $C^{r}$ may be empty.)

EXAMPLE. If $X \subset \mathbf{P}^{n}$ is algebraic of degree $d$ then $\mathscr{J}_{X}^{d}=\mathscr{J}_{X}^{\infty}$. A partial converse is Theorem (17).

The tangent cone $\left.T Y \subset T J\right|_{Y}$ of a subscheme $Y \subset J$ is the locus of tangent vectors annihilating the ideal of $Y$. In particular

$$
T C_{p}^{r}=\left.\left(\operatorname{ker} \pi_{*}\right) \cap T C^{r}\right|_{C_{p}^{r}}
$$

where $C_{p}^{r}=\pi^{-1}(p) \cap C^{r}$ and $\pi_{*}: T J \rightarrow \pi^{*} T \mathbf{P}$ is the differential.

$\pi C_{p}^{r} \subset \mathscr{U}$ is a cone with vertex at $p$. Identify $T_{p, l} C_{p}^{r}$ with the corresponding plane in $\mathbf{P}^{n}$ tangent to $\pi C_{p}^{r}$ along $l$. In local coordinates (9) $T_{p, l} C_{p}^{r}$ is the plane through $x(p)$ cut out by the hyperplanes

$$
\sum \frac{\partial g^{s}(x(p) ; t)}{\partial t_{i}}\left(x_{i}-x(p)\right)=0, \quad s=1, \ldots, r, g \in \mathscr{I}_{X} .
$$


Over a dense open subset $\mathscr{W} \subset C^{r}$ the fibers $T_{p, l} C_{p}^{r}$ will have locally constant dimension. We shall say that $T_{p, l} C_{p}^{r}$ is locally constant along $l$ if $\lambda^{-1}(l) \cap \mathscr{W}$ is nonempty and $T_{p, l} C_{p}^{r}$ is locally constant as a plane in $\mathbf{P}^{n}$ along $\lambda^{-1}(l) \cap \mathscr{W}$.

It is easy to write down the condition for this to happen, using the coordinates of (9) (for a coordinate-free method, see [1, p. 10] "second fundamental form"). Let $(\bar{x}, \bar{t})$ represent $(p, l) \in \lambda^{-1}(l) \cap \mathscr{W} . \lambda^{-1}(l)$ is locally parametrized by $s \mapsto(\bar{x}+s \bar{t}, \bar{t})$ for $s$ near 0 . Regarding $T_{p, l} C_{p}^{r}$ as a subspace of $\mathbf{C}^{n+1}$ we have a vector bundle $T_{;, l} C$. over $\pi^{-1}(l) \cap \mathscr{W}$. Let $v(s)=\sum a_{i}(s) \partial / \partial t_{i}$ be a local holomorphic section, so that

$$
0 \equiv \sum_{i} a_{i}(s) \frac{\partial g^{\nu}}{\partial t_{i}}(\bar{x}+s \bar{t}, \bar{t}), \quad \text { for all } g \in \mathscr{I}_{X}, \nu=1, \ldots, r,
$$

identically in $s . T_{p, l} C_{p}^{r}$ is locally constant along $l$ iff for all such sections $v$ the derivative

$$
0 \equiv \sum_{i} a_{i}^{\prime}(s) \frac{\partial g^{\nu}}{\partial t_{i}}(\bar{x}+s \bar{t}, \bar{t}), \quad \nu=1, \ldots, r, g \in \mathscr{I}_{X},
$$

also vanishes identically.

13. ThEOREM. Fix $r \geq 1$. Suppose

$$
Z \subset\left\{(p, l) \in C^{r} \mid T_{p, l} C_{p}^{r-1}=T_{p, l} C_{p}^{r}\right\}
$$

is a nonempty subscheme, and $\pi \mathrm{Z}$ contains an irreducible component of $\pi C^{r-1}$ as a subscheme. Then

(i) $Z \subset C^{\infty}$,

(ii) $T_{p, l} C_{p}^{r-1}$ is locally constant along the rulings l for generic $(p, l) \in Z$.

Proof. We work in the coordinates (9). Let $(\bar{x}, \bar{t})=(p, l) \in Z, v=$ $\sum a_{i} \partial / \partial x_{i}+\sum b_{i} \partial / \partial t_{i}$. Then $v \in T_{p, l} C^{r-1}$ iff for all $g \in \mathscr{I}_{p, X}, \quad \nu=$ $0, \ldots, r-1$,

$$
0=d g^{\nu}(v)=\sum_{i} a_{i} \frac{\partial g^{\nu}}{\partial x_{i}}+\sum_{i} b_{i} \frac{\partial g^{\nu}}{\partial t_{i}}=\sum_{i} a_{i} \frac{\partial g^{\nu+1}}{\partial t_{i}}+\sum_{i} b_{i} \frac{\partial g^{\nu}}{\partial t_{i}} .
$$

By the Euler relation, $\nu g^{\nu}(\bar{x}, \bar{t})=\sum_{i} \bar{t}_{i} \partial g^{\nu} / \partial t_{i}$. Since $(p, l) \in C^{r}, T_{p, l} C^{r-1}$ contains $w_{x}=\sum \bar{t}_{i} \partial / \partial x_{i}(11)$.

Since $\pi Z$ contains a component of $\pi C^{r-1}$, and $Z \subset C^{r} \subset C^{r-1}$, it follows that at a generic point $(p, l) \in Z$ the differential $\pi_{*}: T_{p, l} C^{r} \rightarrow$ $\pi_{*} T_{p, l} C^{r-1}$ is surjective. Its kernel is $T_{p, l} C_{p}^{r}$. But $T_{p, l} C_{p}^{r}=T_{p, l} C_{p}^{r-1}$, so $T_{p, l} C^{r}=T_{p, l} C^{r-1}$. In particular $w_{x} \in T C^{r}$, so $0=\sum \bar{t}_{i} \partial g^{r} / \partial x_{i}=$ $\sum \bar{t}_{i} \partial g^{r+1} / \partial t_{i}=(r+1) g^{r+1}(\bar{x}, \bar{t})$. Hence $Z \subset C^{r+1}$. 
Now let $v_{t}=\sum b_{i} \partial / \partial t_{i} \in T_{p, l} C_{p}^{r-1}$ be any vector and set $v_{x}=$ $\sum b_{i} \partial / \partial x_{i}$. Then $v_{t} \in T_{p, l} C_{p}^{r}$, hence for all $g \in \mathscr{I}_{p, X}, 0=\sum b_{i} \partial g^{\nu} / \partial t_{i}$, $\nu=1, \ldots, r$. Thus $v_{x} \in T_{p, l} C^{r-1}$. But $T_{p, l} C^{r-1}=T_{p, l} C^{r}$, so $v_{x} \in T_{p, l} C^{r}$, thus $v_{t} \in T_{p, l} C_{p}^{r+1}$. Therefore $T_{p, l} C_{p}^{r}=T_{p, l} C_{p}^{r+1}$ and (i) follows by induction.

As for (ii), if $s \mapsto(\bar{x}+s \bar{t}, \bar{t})$ is a local parametrization of $\lambda^{-1}(l)$ and $\sum a_{i}(s) \partial / \partial t_{i}$ is a local holomorphic section of $T_{,, l} C^{r-1}$ over $\lambda^{-1}(l)$ then

$$
\begin{aligned}
0 & \equiv \sum a_{i}(s) \frac{\partial g^{\nu}}{\partial t_{i}}(\bar{x}+s \bar{t}, \bar{t}), \text { hence } \\
0 & \equiv \sum_{i} a_{i}^{\prime} \frac{\partial g^{\nu}}{\partial t_{i}}+\sum_{i j} a_{i} \bar{t}_{j} \frac{\partial^{2} g^{\nu}}{\partial t_{i} \partial x_{j}}, \quad \nu=1, \ldots, r-1,
\end{aligned}
$$

but $\partial^{2} g^{\nu} / \partial t_{i} \partial x_{j}=\partial^{2} g^{\nu+1} / \partial t_{i} \partial t_{j}$, so the second term vanishes by the Euler relation since $T_{p, l} C_{p}^{r-1}=T_{p, l} C_{p}^{r}$.

REMARK. If $X$ is ruled then the hypotheses of (13) are satisfied for some $r$.

EXAMPLE. Fundamental Forms. (See [4, p. 373].) In affine coordinates, the $r$ th osculating space $T_{p}^{r} X \subset \mathbf{P}^{n}$ is the span of $p$ and the derivatives $\sigma^{\prime}(p), \ldots, \sigma^{(r)}(p)$ of all open curves $\sigma \subset X$ through $p$. Let $p \mapsto \gamma^{r}(p)=$ $T_{p}^{r} X$ be the associated $r$ th order Gauss map. There is a natural way of representing its derivative at a generic point $p$ by an element

$$
d \gamma^{r}(p) \in H^{0}\left(\mathbf{P} T_{p} X, \mathcal{O}(r+1)\right) \otimes N_{p}\left(T_{p}^{r} X\right)
$$

where $N\left(T_{p}^{r} X\right)=T_{p} \mathbf{P}^{n} / T_{p}\left(T_{p}^{r} X\right)$ is the normal space. $d \gamma^{r}(p)$ is the $r+1$ st fundamental form of $X$ at $p$.

Let $v=\sum a_{i, \sigma} \sigma^{(i)}$ be any local section of the associated bundle $T^{r} X$ (with fiber $\left.\left(T^{r} X\right)_{q}=T_{q}^{r} X\right)$ defined near $p$. Then

$$
v^{\prime}(p) \equiv \sum a_{r, \sigma} \sigma^{(r+1)} \bmod T_{p}\left(T_{p}^{r} X\right)
$$

in coordinates. So define $d \gamma^{r}$ by

$$
\left.\left[d \gamma^{r}\left(\sigma^{\prime}(p)^{\otimes r+1}\right)\right]\right\lrcorner d g=(g \circ \sigma)^{(r+1)}(p), \quad \text { for all } g \in \mathscr{I}_{T_{p}^{r} X, p} .
$$

(This does not depend on any choices.)

The associated linear system

$$
\left.L^{r+1}=\left\{d \gamma^{r}\right\lrcorner \theta \mid \theta \in N_{p}^{*}\left(T_{p}^{r} X\right)\right\} \subset H^{0}\left(\mathbf{P} T_{p} X, \mathcal{O}(r+1)\right)
$$

is contained in the ideal of $C_{p}^{r+1}$ (viewed as a subvariety of $\mathbf{P} T_{p} X$ ). (Since $p$ is a generic point we may represent $X$ as a graph $y_{j}=f_{j}(x), j=1, \ldots, k$, $x=\left(x_{1}, \ldots, x_{m}\right)$ in affine coordinates near $p$. If $g=\sum a_{j} y_{j}$ vanishes on 
$T_{p}^{r} X$ then $\left.d \gamma^{r}\right\lrcorner d g=\sum a_{j} f_{j}^{r+1}(x(p) ; d x)$. For $r \geq 1$ this is the $r+1$ st order part of an element, $\sum a_{j}\left(f_{j}(x)-y_{j}\right)$, of $\left.\mathscr{I}_{X}\right)$. Geometrically the reason is that, if $(p, l) \in C_{p}^{r+1}$ then choose a curve $\sigma \subset X$ through $p$ which meets $l$ through order $r+1 . \sigma^{\prime}(p), \ldots, \sigma^{(r+1)}(p)$ lie along $l \subset$ $T_{p}^{1} X \subset T_{p}^{r} X$, so if $g$ vanishes on $T_{p}^{r} X$ then $(g \circ \sigma)^{(r+1)}(p)=0$.

At a generic $p, L^{2}$ generates the ideal of $C_{p}^{2}$ in $\mathbf{P} T_{p} X$, but this is not in general true of the higher $L^{r}$ 's. For example, if $X$ is a hypersurface, not a hyperplane, then $T_{p}^{2} X=\mathbf{P}^{n}$ so $L^{3}=\{0\}$. But $\mathscr{J}_{X}^{3} \neq \mathscr{J}_{X}^{2}$ unless $X$ is ruled (12). A less trivial example is the following, due to Mark Green:

EXAmple. (Green [3].) Consider the surface $X \subset \mathbf{P}^{4}$ parametrized by

$$
p(s, t)=\left(t, s^{2} t^{2}, s^{6} t^{3}, s^{12} t^{4}\right)
$$

in affine coordinates. Then

$$
\frac{\partial^{2} p}{\partial t^{2}}=\frac{s}{t^{2}} \frac{\partial p}{\partial s}
$$

so every $Q \in L^{2}$ vanishes on $(\partial p / \partial t)^{\otimes 2}$. In fact

$$
L^{2}=\operatorname{span}\left\{d s^{2}, d s \cdot d t\right\} \text {. }
$$

By a result of Griffiths and Harris [4, p. 373], the Jacobian system of $L^{r+1}$ is contained in $L^{r}, r=2,3, \ldots$ It follows that

$$
L^{r} \equiv 0 \quad \bmod \left\{d s^{r}, d s^{r-1} \cdot d t\right\}, \quad r=2,3, \ldots
$$

Griffiths and Harris conjectured that any surface with such $L^{r}$ 's ought to be ruled [4, p. 377]. But $X$ is not ruled. In particular, by (12), the $L^{r}$ 's cannot generate the ideal of $C_{p}^{r}$ if $r \geq 3$ at a generic $p$.

EXAMPLE. [4, p. 387]. The second fundamental form represents the derivative of the Gauss map $\gamma=\gamma^{1}$. $\operatorname{ker} d \gamma_{p}$ (projectivized) is the common singular locus in $\mathbf{P} T_{p} X$ of all the quadrics in $L^{2}$.

Conversely if, at a generic $p \in X$, all the quadrics in $L^{2}$ have a common singular locus $Z_{p}$, then the hypotheses of (13) are satisfied with $r=2$ : take $Z=\cup Z_{p}$. Then $X$ is ruled by the planes $\pi Z_{p}$, which are the fibers of $\gamma$ (locally).

Examples of such $X$ are cones and developable varieties. Recently F. Zak [7, p. 540 see [2] for a proof] proved that if $X$ is a smooth algebraic variety of degree $\geq 2$ then the fibers of $\gamma$ are finite (zero dimensional).

14. Corollary. Let $X \subset \mathscr{U}$ be an irreducible variety. If $X$ is not ruled then over a generic $p \in X$ the dimensions $\operatorname{dim} T_{p, l} C_{p}^{r}, r=0,1,2, \ldots$, are strictly decreasing to zero for all $(p, l) \in \pi^{-1}(p)$. 
Proof. Let $Z^{r}=\left\{(p, l) \in C^{r} \mid T_{p, l} C_{p}^{r}=T_{p, l} C_{p}^{r-1}\right\} . Z^{r}$ is an analytic variety. Since $\pi$ is proper, $\pi Z^{r}$ is an analytic subvariety of $X$. If $X$ is the countable union $X=\bigcup_{r=1}^{\infty} \pi Z^{r}$ then one of the $Z^{r}$ 's, say $Z^{r}$, must map dominantly to $X$. Restricting to an open subset one may assume $Z^{r}$ is surjective. Then $X=\pi Z^{r}=\pi C^{r-1}$. Apply (13).

The following answers a question in Griffiths and Harris [4, p. 450].

15. Corollary. Let $X \subset \mathscr{U}$ be an irreducible hypersurface, $p \in X a$ generic point. Then for each $r=1, \ldots, n=\operatorname{dim} \mathbf{P}^{n}$, if $C_{p}^{s}$ is not a smooth complete intersection of type $(1,2, \ldots, s)$ in $\mathbf{P}\left(T_{p} \mathbf{P}^{n}\right)$ for all $s=1, \ldots, r$ (if $s=n$ this means $C_{p}^{n}$ is not empty) then $X$ is ruled, and $C_{p}^{r}$ is singular or has codimension $<r$ in $\mathbf{P}\left(T_{p} \mathbf{P}^{n}\right)$.

Proof. Let $g$ be a local generator for $\mathscr{I}_{X}$. Then $C_{p}^{r} \cong\left\{t \mid g^{1}(x(p) ; t)\right.$ $\left.=\cdots=g^{r}(x(p) ; t)=0\right\}$ in $\mathbf{P}\left(T_{p} \mathbf{P}^{n}\right)$. Let $1 \leq r \leq n$ be the least integer such that $C_{p}^{r}$ is not a smooth complete intersection of type $(1, \ldots, r)$. Then $C_{p}^{r}$ is singular or $C_{p}^{r}=C_{p}^{r-1}$. Since $C_{p}^{r}$ has codimension at most 1 in $C_{p}^{r-1}$ it follows that for some $(p, l) \in C_{p}^{r}, T_{p, l} C_{p}^{r}=T_{p, l} C_{p}^{r-1}$. Apply (14).

If $X$ is ruled then say the rulings are in general position if $\left(\operatorname{span} C_{p}^{\infty}\right)$ $=\mathbf{P} T_{p} X$ at a generic $p \in X$.

16. LeMMA. Let $\mathscr{U} \subset \mathbf{P}^{n}$ be an open set, $X \subset \mathscr{U}$ an irreducible, ruled variety whose rulings are in general position. Then $X$ is piecewise linearly connected i.e. given $p, q \in X$ there exists a finite sequence $l_{i}, i=0, \ldots, m$, of line segments in $X$ such that $p \in l_{0}, q \in l_{m}$ and $l_{i}$ meets $l_{i+1}$ for each $i$.

Proof. Let $Y \subset X$ be the locus of points $p \in X$ such that $C_{p}^{\infty}$ spans $T_{p} X . Y$ is a dense open subset. Let $\mathscr{U}^{\prime} \subset \mathscr{U}$ be a convex open subset such that $\mathscr{U}^{\prime} \cap X \subset Y$ is nonempty. Let $X^{\prime} \subset \mathscr{U}^{\prime}$ be an irreducible component of $\mathscr{U}^{\prime} \cap X$, and let $C^{\infty \prime}$ be the $\infty$ contact cone of $X^{\prime}$ in $\pi^{-1} \mathscr{U}^{\prime}$. Let $p^{\prime} \in X^{\prime}$.

Since $\pi: \pi^{-1} \mathscr{U}^{\prime} \rightarrow \mathscr{U}^{\prime}$ is a proper map one can define a sequence of analytic subvarieties of $X$ by

$$
C_{p^{\prime}}^{\infty \prime}(1)=\pi C_{p^{\prime}}^{\infty \prime}, \quad C_{p^{\prime}}^{\infty \prime}(k+1)=\pi \pi^{-1} C_{p^{\prime}}^{\infty}(k), \quad k=1,2,3, \ldots
$$

Clearly $C_{p^{\prime}}^{\infty}(k+1)$ consists of all points in $X^{\prime}$ connected to points in $C_{p^{\prime}}^{\infty}(k)$ by line segments in $X^{\prime}$. Eventually the dimension of $C_{p^{\prime}}^{\infty \prime}(k)$ will reach a maximum. Then a generic smooth point $q^{\prime}$ of $C_{p^{\prime}}^{\infty}(k)$ is also a smooth point of $C_{p^{\prime}}^{\infty}(k+1)$. But $C_{p^{\prime}}^{\infty \prime}(k+1)$ contains all the lines in $X^{\prime}$ 
through $q^{\prime}$. Since the rulings are in general position, $\operatorname{dim} C_{p^{\prime}}^{\infty \prime}(k+1)=$ $\operatorname{dim} X^{\prime}$. Since $X^{\prime}$ is irreducible, $C_{p^{\prime}}^{\infty}(k+1)=X^{\prime}$.

Now replace $X^{\prime}$ by $X, \mathscr{U}^{\prime}$ by $\mathscr{U}$. Going through the same construction, construct $C_{p^{\prime}}^{\infty \prime}(k+1)$. Then $C_{p^{\prime}}^{\infty \prime}(k+1) \subset C_{p^{\prime}}^{\infty}(k+1)$; since $X$ is irreducible, $C_{p^{\prime}}^{\infty}(k+1)=X$. So every point $p \in X$ can be connected to $p^{\prime}$ by at most $k+1$ line segments, hence any two points can be connected to each other by at most $2 k+2$ line segments.

17. TheOREM. Let $X \subset \mathscr{U} \subset \mathbf{P}^{n}$ be an irreducible analytic hypersurface, $p \in X, g \in \mathscr{I}_{p, X}$ a generator. Assume

(i) $\mathscr{J}_{X}^{d}=\mathscr{J}_{X}^{d+1}$ for some $d \leq n-1$.

(ii) $g^{1}(x(p) ; t), \ldots, g^{d}(x(p) ; t)$ are a regular sequence of polynomials

(iii) $C_{p}^{d}$ is reduced.

Then $X$ is algebraic-there is a polynomial $f\left(x_{1}, \ldots, x_{n}\right)$ of degree $\leq d$ (in affine coordinates) vanishing on $X$.

Proof. Recall some consequences of (i), (ii), (iii):

18. $C_{p}^{d}=\left\{t \in \mathbf{P} T_{p} \mathbf{P}^{n} \mid g^{1}(x(p) ; t)=\cdots=g^{d}(x(p) ; d)=0\right\}, g \in$ $\mathscr{I}_{X, p}$ a generator, is nonempty (since $d \leq n-1$ ), smooth on a dense open subset (by (iii)), and $C_{p}^{d}=C_{p}^{\infty}$ (by (12)).

19. Every homogeneous polynomial vanishing identically on $C_{p}^{d}$ is in the homogeneous ideal generated by $g^{1}, \ldots, g^{d}$.

20. Every homogeneous relation $\sum_{r=1}^{d} a^{r} g^{r}=0$ is of the form $a^{r}=$ $\sum_{s} Q_{r s} g^{s}$ where $Q_{r s}$ is an antisymmetric matrix of polynomials $(19,20$ follow from (ii), (iii); use a Koszul complex).

21. If $a^{i}(t), i=1, \ldots, d$, are homogeneous polynomials satisfying the identity

$$
0 \equiv \sum_{i=1}^{d} a^{i} \frac{\partial g^{i}}{\partial t_{\lambda}} \bmod g^{1}, \ldots, g^{d}, \quad \text { for all } \lambda=1, \ldots, n,
$$

then $a^{i} \equiv 0 \bmod g^{1}, \ldots, g^{d}$, for all $i$.

Proof of 21. If $\sum_{i=1}^{d} a^{i} d g^{i} \equiv 0 \bmod g^{1}, \ldots, g^{d}$ then $0 \equiv \sum_{i=1}^{d} a^{i} d g^{i} \wedge$ $d g^{1} \wedge \cdots \wedge \widehat{d g}^{j} \wedge \cdots \wedge d g^{d} \equiv \pm a^{j} d g^{1} \wedge \cdots \wedge d g^{d} \bmod g^{1}, \ldots, g^{d}$. By $18, d g^{1} \wedge \cdots \wedge d g^{d} \neq 0$ on a dense open subset of $C_{p}^{d}$, so $a^{j} \equiv 0$ on $C_{p}^{d}$. Apply 19. 
22. The points of $C_{p}^{d}$ are in general position in the hyperplane $g^{1}(t)=0$ (by 19, since $\left.\operatorname{deg} g^{i}=i\right)$.

Proof of theorem. We may assume $g$ generates $\mathscr{I}_{X}$ on $\mathscr{U}$. Taken together (ii), (iii) are open conditions-assume they are satisfied everywhere on $\mathscr{U}$. We shall work in the ring $\mathcal{O}_{\mathscr{U}}[t]$ of polynomials in $t$ with holomorphic coefficients. All polynomials are homogeneous. Degree means degree as a polynomial in $t$.

Set $e=d+1$. As in the proof of (12) one has local relations on $\pi^{-1} \mathscr{U}$ :

$$
\begin{gathered}
0 \equiv \sum_{i=0}^{e} a^{e-i}(x ; t) g^{i}(x ; t), \\
0 \equiv \sum_{i=0}^{e+1} b^{e+1-i}(x ; t) g^{i}(x ; t) .
\end{gathered}
$$

$\operatorname{deg} a^{i}=\operatorname{deg} b^{i}=i$ for all $i$, and $a^{0}, b^{0} \neq 0$. The idea is this: if $f(x)=$ $g(x) h(x)$ were a polynomial of degree $<e$ (in $x$ ) vanishing on $X$ then, expanding as a power series, one has $0 \equiv f^{e}=\sum h^{e-i} g^{l}$. So one can hope to recover $f$ from (23).

One may replace $b^{i}$ by $b^{i}\left(a^{0} / b^{0}\right)+a^{i-1}\left(a^{1} / a^{0}-b^{1} / b^{0}\right), \quad i=$ $0, \ldots, e+1,\left(\right.$ set $\left.a^{-1}=0\right)$. Then

$$
a^{0}=b^{0}, \quad a^{1}=b^{1} .
$$

Differentiate (23) with respect to $x_{\lambda}$ and (24) with respect to $t_{\lambda}$ :

$$
\begin{aligned}
& 0 \equiv \sum_{i=0}^{e} \frac{\partial a^{e-i}}{\partial x_{\lambda}} g^{i}+\sum_{i=0}^{i} a^{e-i} \frac{\partial g^{i}}{\partial x_{\lambda}} \equiv \sum_{i=0}^{e} \frac{\partial a^{e-i}}{\partial x_{\lambda}} g^{i}+\sum_{i=1}^{e+1} a^{e+1-i} \frac{\partial g^{i}}{\partial t_{\lambda}} \\
& 0 \equiv \sum_{i=0}^{e} \frac{\partial b^{e+1-i}}{\partial t_{\lambda}} g^{i}+\sum_{i=1}^{e+1} b^{e+1-i} \frac{\partial g^{i}}{\partial t_{\lambda}}
\end{aligned}
$$

for all $\lambda=1, \ldots, n$, since $\operatorname{deg} g^{0}=\operatorname{deg} b^{0}=0$. Subtract:

$$
0 \equiv \sum_{i=0}^{e}\left(\frac{\partial a^{e-i}}{\partial x_{\lambda}}-\frac{\partial b^{e+1-i}}{\partial t_{\lambda}}\right) g^{i}+\sum_{i=1}^{e-1}\left(a^{e+1-i}-b^{e+1-i}\right) \frac{\partial g^{i}}{\partial t_{\lambda}}
$$

Since $g^{1}, \ldots, g^{e-1}$ is a regular sequence it follows (21) that $a^{e+1-\imath} \equiv$ $b^{e+1-i} \bmod g^{0}, \ldots, g^{e-1}$ for all $i=1, \ldots, e+1$. Define $a^{e+1}=b^{e+1}$. Write

$$
b^{e+1-i}=a^{e+1-i}+\sum_{j=0}^{e+1} P_{i j} g^{j}, \quad i=0, \ldots, e+1,
$$


where $P_{i j}$ has degree $e+1-i-j$ when $0 \leq i, j, i+j \leq e+1$ and vanishes for $i, j$ outside this range. Set

$$
A_{i j}=\sum_{r=0}^{j+1} P_{i+1+r, j-r}-\sum_{r=0}^{i+1} P_{j+1+r, i-r}
$$

Then $A_{i j}=-A_{j i}, \operatorname{deg} A_{i j}=e-i-j$ for all $i, j$, and

$$
A_{i-1, j}-A_{i, j-1}=P_{i j}+P_{j i} \text { for all } i, j=0, \ldots, e+1 \text {. }
$$

Define $B_{i j}$ by

$$
A_{i-1, j}+A_{i, j-1}-2 B_{i j}=P_{i j}-P_{j i}, \quad i, j=0, \ldots, e+1 .
$$

Then $B_{i j}=-B_{j i}, \operatorname{deg} B_{i j}=e+1-i-j$ for all $i, j$. Set

$$
\begin{aligned}
\bar{a}^{e-i} & =a^{e-i}+\sum_{j=0}^{e+1} A_{i j} g^{j}, & & i=-1, \ldots, e, \\
\bar{b}^{e+1-i} & =b^{e+1-i}+\sum_{j=0}^{e+1} B_{i j} g^{j}, & i & =0, \ldots, e+1 .
\end{aligned}
$$

Since $A_{i j}, B_{i j}$ are antisymmetric the $\bar{a}^{i}, \bar{b}^{i}$ satisfy $(23,24)$. Moreover they have the right degree, and $\bar{a}^{0}=a^{0}+A_{e o} g^{0}$ does not vanish near the locus $\left(g^{0}=0\right)$. Finally, one may check using (26), that

$$
\bar{a}^{i}=\bar{b}^{i}, \quad i=0, \ldots, e+1 .
$$

Replace the $a, b$ 's by the $\bar{a}, \bar{b}$ 's in $(23,24)$. Then (25) becomes

$$
0=\sum_{i=0}^{e}\left(\frac{\partial a^{e-i}}{\partial x_{\lambda}}-\frac{\partial a^{e+1-i}}{\partial t_{\lambda}}\right) g^{i}, \quad \lambda=1, \ldots, n .
$$

Subtract $\left(\partial a^{0} / \partial x_{\lambda}-\partial a^{1} / \partial t_{\lambda}\right) / a^{0}$ times eq. (23) from this and get

$$
0 \equiv \sum_{i=0}^{e-1}\left\{\frac{\partial a^{e-1}}{\partial x_{\lambda}}-\frac{\partial a^{e+1-i}}{\partial t_{\lambda}}-\frac{a^{e-i}}{a^{0}}\left(\frac{\partial a^{0}}{\partial x_{\lambda}}-\frac{\partial a^{1}}{\partial t_{\lambda}}\right)\right\} g^{i}
$$

a homogeneous relation among the $g^{i}$ 's. Reducing $\bmod g^{0}$ one can apply (20), then by adding an appropriate multiple of $g^{0}$ one has

$$
\frac{\partial a^{e-i}}{\partial x_{\lambda}}=\frac{\partial a^{e+1-i}}{\partial t_{\lambda}}+\frac{a^{e-i}}{a^{0}}\left(\frac{\partial a^{0}}{\partial x_{\lambda}}-\frac{\partial a^{1}}{\partial t_{\lambda}}\right)+\sum_{j=0}^{e-1} Q_{i j}^{\lambda} g^{j}
$$

$i=0, \ldots, e-1, \lambda=1, \ldots, n, \operatorname{deg} Q_{i j}^{\lambda}=e-i-j$ where $Q_{i j}^{\lambda}$ is an antisymmetric matrix of polynomials.

Multiplying (23) by $1 / a^{0}$ we may assume $a^{0} \equiv 1$. Then for $i=e-1$ (27) becomes

$$
\frac{\partial a^{1}}{\partial x_{\lambda}}=\frac{\partial a^{2}}{\partial t_{\lambda}}-a \frac{\partial a^{1}}{\partial t_{\lambda}}+Q_{e-1,0}^{\lambda} g^{0}+Q_{e-1,1}^{\lambda} g^{1}
$$


Consider the form

$$
\Phi=\sum_{\mu} \frac{\partial a^{1}}{\partial t_{\mu}} d x_{\mu}, \quad d \Phi=\sum_{\lambda \mu} \frac{\partial^{2} a^{1}}{\partial x_{\lambda} d t_{\mu}} d x_{\lambda} \wedge d x_{\mu} .
$$

Applying (28),

$$
d \Phi=g^{0} \sum_{\lambda \mu} \frac{\partial Q_{e-1,0}^{\lambda}}{\partial t_{\mu}} d x_{\lambda} \wedge d x_{\mu}+\sum_{\lambda \mu} Q_{e-1,1}^{\lambda} \frac{\partial g^{1}}{\partial t_{\mu}} d x_{\lambda} \wedge d x_{\mu} .
$$

Since $\sum\left(\partial g^{1} / \partial t_{\mu}\right) d x_{\mu}=d g^{0}, \Phi$ is closed along $\left(g^{0}=0\right)$. So locally along $\left(g^{0}=0\right)$ one can solve the equation $d \log h(x)=\Phi$. Multiply the $a^{i}$ 's by $h(x)$. Then

$$
\sum_{\lambda} \frac{\partial a^{0}}{\partial x_{\lambda}} d x_{\lambda}=\sum_{\lambda} \frac{\partial a^{1}}{\partial t_{\lambda}} d x_{\lambda} \quad \bmod g^{0}, d g^{0} .
$$

Let $\bar{x} \in X$. Define a polynomial $f(x)$ of degree $\leq e-1$ by

$$
f(x)=\sum_{i=0}^{e-1} \sum_{j=0}^{i} a^{i-j}(\bar{x}, x-\bar{x}) g^{j}(\bar{x}, x-\bar{x}) .
$$

It remains to show that $f$ vanishes on $X$. Clearly

$$
\begin{aligned}
& 0=f^{0}(\bar{x})=a^{0}(\bar{x}) g^{0}(\bar{x}), \\
& f^{r}(\bar{x} ; t)=\sum_{j=0}^{r} a^{r-j}(\bar{x} ; t) g^{j}(\bar{x} ; t), \quad r=0, \ldots, e-1, \quad \text { and } \\
& f^{e}(x ; t) \equiv 0 .
\end{aligned}
$$

Define functions

$$
\begin{array}{r}
f_{\lambda}^{r}(x ; t)=\sum_{j=0}^{r-1} \frac{\partial a^{r-j}}{\partial t_{\lambda}}(x ; t) g^{j}(x ; t)+\sum_{j=1}^{r} a^{r-j}(x ; t) \frac{\partial g^{j}}{\partial t_{\lambda}}(x ; t), \\
r=1, \ldots, e, \lambda=1, \ldots, n .
\end{array}
$$

In particular $f_{\lambda}^{e} \equiv 0$ by (23), and $f_{\lambda}^{r}$ is homogeneous of degree $r-1$. Differentiate:

$$
\frac{\partial f_{\mu}^{r}}{\partial x_{\lambda}}-\frac{\partial f_{\mu}^{r+1}}{\partial t_{\lambda}} \equiv \sum_{j=1}^{r}\left(\frac{\partial a^{r-j}}{\partial x_{\lambda}}-\frac{\partial a^{r+1-j}}{\partial t_{\lambda}}\right) \frac{\partial g^{j}}{\partial t_{\mu}} \bmod g^{0}, \ldots, g^{e-1}
$$

Then substituting in $(27,29)$ this becomes

$$
\sum_{\lambda}\left(\frac{\partial f_{\mu}^{r}}{\partial x_{\lambda}}-\frac{\partial f_{\mu}^{r+1}}{\partial t_{\lambda}}\right) d x_{\lambda} \equiv 0 \quad \bmod g^{0}, \ldots, g^{e-1}, d g^{0} .
$$


Let $l(s)=(x+s t, t)$ be a line in $C^{e-1}$. Then $g^{0}, \ldots, g^{e-1}$ vanish on l. So by (32)

$$
\left.\frac{d}{d s}\right|_{0} f_{\mu}^{r}(x+s t, t)=\sum_{\lambda} t_{\lambda} \frac{\partial f_{\mu}^{r}}{\partial x_{\lambda}}(x, t)=r f_{\mu}^{r+1}(x, t),
$$

along $l$. Now it is easy to show that functions $f_{\mu}^{r}$, homogeneous of degree $r-1$ in $t$, satisfying (33) and the condition $f_{\mu}^{e} \equiv 0$ are uniquely determined along a line by their values at a single point.

On the other hand the functions $\left(\partial f^{r} / \partial t_{\mu}\right)(x ; t)$ derived from the polynomial (30) also satisfy these relations, moreover they agree with the $f_{\mu}^{r}$ 's at any point $(\bar{x}, \bar{t})$ lying on a line in $C^{e-1}$ through $\bar{x}$ (differentiate (31) at $\bar{x}$ ). By (22) the rulings of $X$ are in general position, so by (12), (16) $f_{\mu}^{r}=\partial f^{r} / \partial t_{\mu}$ everywhere on $C^{e-1}$.

In particular $\partial f^{1} / \partial t_{\lambda}=f_{\lambda}^{1}$ on $C^{e-1}$. But $d f^{0}=\sum\left(\partial f^{1} / \partial t_{\lambda}\right) d x_{\lambda}$ and

$$
\sum f_{\lambda}^{1} d x_{\lambda}=\sum\left(\frac{\partial a^{1}}{\partial t_{\lambda}} g^{0}+a^{0} \frac{\partial g^{1}}{\partial t_{\lambda}}\right) d x_{\lambda} \equiv 0 \bmod g^{0}, d g^{0} .
$$

Hence $f^{0}$ is constant $=f^{0}(\bar{x})=0$ on $C^{e-1}$. Since $\pi C^{e-1}=X$ (18), $f$ vanishes on $X$.

EXAMPLE. If $C_{p}^{r}$ is not reduced then the conclusion of (17) may not hold.

Let $X \subset \mathbf{P}^{3}$ be the cylinder

$$
X=\left\{\left(x_{1}, x_{2}, x_{3}\right) \mid g\left(x_{1}, x_{2}\right)=0\right\}
$$

in affine coordinates. $X$ may not be algebraic (if $g$ is not).

$$
\begin{aligned}
& g^{1}(x ; d x)=g_{1} d x_{1}+g_{2} d x_{2} \\
& g^{2}(x ; d x)=\frac{1}{2}\left(g_{11} d x_{1}^{2}+2 g_{12} d x_{1} d x_{2}+g_{22} d x_{2}^{2}\right)
\end{aligned}
$$

etc., where $g_{t}=\partial g / \partial x_{i}$. If $g^{1}(x(p) ; d x) \neq 0$ and $g^{1}(x(p) ; d x)$ does not divide $g^{2}(x(p) ; d x)$ then $g^{1}, g^{2}$ are a regular sequence generating any homogeneous cubic in $d x_{1}, d x_{2}$. In particular $g^{3} \equiv 0 \bmod g^{1}, g^{2} . C_{p}^{2}$ is supported on the point $\left[d x_{1}, d x_{2}, d x_{3}\right]=[0,0,1]$ but it is not reduced, since $\left\{d x_{1}, d x_{2},\right\} \not \subset \mathscr{J}_{X}^{2}$.

I would like to thank my teachers at UCLA, especially Mark Green, for their invaluable help and encouragement.

\section{REFERENCES}

[1] A. Altman and S. Kleiman, Introduction to Grothendieck Duality Theory, Springer Lecture Notes 146, 1970.

[2] W. Fulton and R. Lazersfeld, Connectivity and its applications in algebraic geometry, (preprint), Brown University. 
[3] Mark Green (Lecture given at UCLA, 1983).

[4] P. Griffiths and J. Harris, Algebraic geometry and local differential geometry, Ann. Scient. Éc. Norm. Sup. $4^{e}$ série, t. 12 (1979), 355-432.

[5] A. Grothedieck and J. Dieudonné, Eléments de Géométrie Algébrique IV, Publ. Math. I.H.E.S., 32 (1967).

[6] R. Hartshorne, Algebraic Geometry, Graduate Texts in Math., Springer-Verlag, 1977.

[7] F. Zak, Projection of algebraic varieties, Math. USSR Sbornik, 44 (1983), 535-544.

Received June 3, 1985 and in revised form October 20, 1985.

UNIVERSITY OF WASHINGTON

SEATTLE, WA 98195 



\section{PACIFIC JOURNAL OF MATHEMATICS EDITORS}

\author{
V. S. VARADARAJAN \\ (Managing Editor) \\ University of California \\ Los Angeles, CA 90024 \\ HERBERT ClEMENS \\ University of Utah \\ Salt Lake City, UT 84112 \\ R. FINN \\ Stanford University \\ Stanford, CA 94305
}

\author{
HERMANN FLASCHKA \\ University of Arizona \\ Tucson, AZ 85721 \\ RAMESH A. GANGOLLI \\ University of Washington \\ Seattle, WA 98195 \\ VAUghan F. R. JONES \\ University of California \\ Berkeley, CA 94720 \\ ROBION KIRBY \\ University of California \\ Berkeley, CA 94720
}

C. C. MOORE

University of California Berkeley, CA 94720

H. SAMELSON

Stanford University Stanford, CA 94305

HAROLD STARK

University of California, San Diego La Jolla, CA 92093

\section{ASSOCIATE EDITORS}
R. ARENS
E. F. BECKENBACH
B. H. NEUMANN
F. WOLF
K. YOSHIDA (1906-1982)

\section{SUPPORTING INSTITUTIONS}

UNIVERSITY OF ARIZONA

UNIVERSITY OF BRITISH COLUMBIA

CALIFORNIA INSTITUTE OF TECHNOLOGY

UNIVERSITY OF CALIFORNIA

MONTANA STATE UNIVERSITY

UNIVERSITY OF NEVADA, RENO

NEW MEXICO STATE UNIVERSITY

OREGON STATE UNIVERSITY
UNIVERSITY OF OREGON UNIVERSITY OF SOUTHERN CALIFORNIA

STANFORD UNIVERSITY

UNIVERSITY OF HAWAII

UNIVERSITY OF TOKYO

UNIVERSITY OF UTAH

WASHINGTON STATE UNIVERSITY

UNIVERSITY OF WASHINGTON 


\section{Pacific Journal of Mathematics}

Vol. 125, No. 1 September, 1986

Gilles Christol, Fonctions et éléments algébriques $\ldots \ldots \ldots \ldots \ldots \ldots \ldots \ldots$

Jo-Ann Deborah Cohen, Extensions of valuation and absolute valued

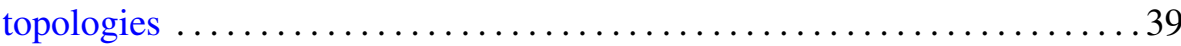

Miriam Cohen, Smash products, inner actions and quotient rings . . . . . . 45

Mikio Furushima, On the singular $K-3$ surfaces with hypersurface

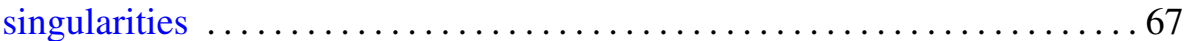

Gerhard Gierz and Boris Shekhtman, A duality principle for rational

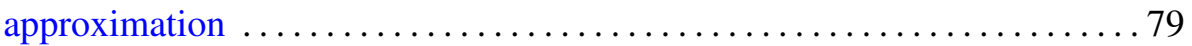

Anthony Wood Hager, A description of HSP-like classes, and

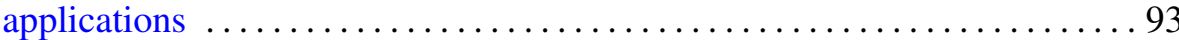

George Alan Jennings, Lines having high contact with a projective

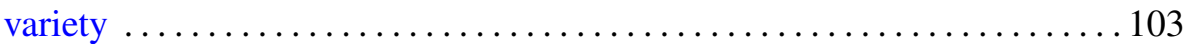

John Lott, Eigenvalue bounds for the Dirac operator . . . . . . . . . . . 117

Denis Laurent Luminet, A functional calculus for Banach PI-algebras . . . . 127

Shizuo Miyajima and Noboru Okazawa, Generators of positive

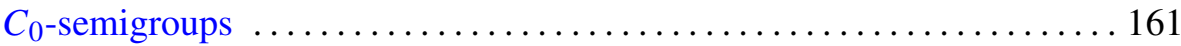

Takemi Mizokami, On functions and stratifiable $\mu$-spaces $\ldots \ldots \ldots \ldots \ldots 177$

Jeff Parker, 4-dimensional $G$-manifolds with 3-dimensional orbits . . . . . 187

Elias Saab and Paulette Saab, On Peł czyński's properties (V) and (V*) . . 205

Elmar Schrohe, The symbols of an algebra of pseudodifferential operators

Aart van Harten and Els Vader-Burger, Approximate Green functions as a tool to prove correctness of a formal approximation in a model of competing and diffusing species

Stephen Watson, Using prediction principles to construct ordered

continua 